\title{
Influence of body weight, age and management system on reproduction of South African Angora goat does
}

\author{
M.A. Snyman \\ Grootfontein Agricultural Development Institute, Private Bag X529, Middelburg (EC) 5900, South Africa
}

\begin{abstract}
The effect of doe age, body weight and different management systems, as practiced in various Angora goat studs, on reproductive performance of does was investigated. The data used were collected from 2000 to 2004 on 12 Angora goat studs kept under different management systems. The data set analysed for this study contained data of 6271 does, each with a varying number of kidding opportunities. This amounted to 14644 doe records where each record included body weight before mating, body weight at scanning (ultrasound scanning for pregnancy diagnosis) and all reproductive performance data. There was a wide range pertaining to reproductive performance among the various studs. Apart from a high kid mortality rate, which is regarded by breeders as the most important factor contributing to low weaning percentages, nearly $20 \%$ of the does did not conceive or lost their foetuses before birth. Body weight of does before mating ranged from $13.0 \mathrm{~kg}$ to $59.6 \mathrm{~kg}$ (average $=34.6 \mathrm{~kg}$ ) among animals, while body weight at scanning ranged from $18.0 \mathrm{~kg}$ to $67.0 \mathrm{~kg}$ (average $=38.6 \mathrm{~kg}$ ). All reproductive parameters recorded had a typical inverted U-shaped relationship with age of dam, where the 2- and 3-year-old does and does older than 9 years of age fared significantly worse than the 4- to 9-year-old does. Management system had a significant influence on reproductive performance of does in the different studs. Higher reproductive rates were recorded under those management systems where additional or supplementary feeding at various stages of the reproductive cycle were supplied. Body weights before mating and at scanning had significant positive relationships with reproduction. For every kilogram increase in body weight before mating, 0.0237 and 0.0218 more kids will be born and weaned, respectively. Body weights before mating of young does that were kept on pastures until 18 months of age were higher than those of maiden does in the other management systems, resulting in higher reproductive rates. Number of kids scanned, born and weaned per maiden doe at first kidding increased with an increase in body weight at first mating. From the results it is evident that body weight, age of the doe and management system all have a significant effect on the reproduction of Angora goats.
\end{abstract}

Keywords: Nutrition, mating, pregnancy, kidding performance

\#E-mail: grethasn@daff.gov.za

\section{Introduction}

Reproductive performance is the most important trait determining income from all livestock enterprises. The relatively low reproductive rate and high kid mortality rate of Angora goats are well documented (Terblanché, 1988; Geyer, 1998). There are numerous internal and external factors that contribute to the actual number of kids born per doe that was put to the ram. There are even more factors which determine whether a kid born alive will survive to weaning age. Age of dam is one of the internal factors that have a marked influence on overall efficiency of the flock (Van der Westhuizen et al., 2004). There is a tendency among Angora goat stud breeders to retain stud does in the breeding flock up to the age of 12 years. Body weight of the doe, and therefore all factors influencing body weight per se, is another important factor contributing to reproductive efficiency. A positive relationship between reproduction and body weight has been reported for sheep (Safari \& Fogarty, 2003) and goats (Constantinou, 1989). The effect of doe age, body weight and different management systems, as practiced in 12 Angora goat studs, on reproductive performance of does will be investigated in this paper.

\section{Material and Methods}

The data used for this part of the study were collected from 2000 to 2004 on 12 Angora goat studs, run under different management systems. The management practices followed in the studs from mating until weaning were grouped into five major management systems, and are summarised in Table 1. 
The data set analysed for this study contained data of 6271 does. Of these does, 392 had five kidding opportunities, 1033 does had four, 1146 does had three, 1414 does had two and 2286 does only had one kidding opportunity during the five years of data recording. This amounted to 14644 doe records, where each record included body weight before mating, body weight at scanning (ultrasound scanning for pregnancy diagnosis), number of kids scanned, born and weaned for each year the doe was present in the flock. Data collected during the kidding seasons included the following information for each doe: Doe identity, sire identity (if known), kid identity, kidding date, birth weight, sex, birth and rearing status, rearing group and weaning weight of her kids. Does that failed to kid, aborted or delivered stillborn kids or does that died before kidding, were also recorded.

Table 1 Management systems (MS) followed in the various studs

\begin{tabular}{|c|c|c|c|c|c|}
\hline $\begin{array}{l}\text { MS } \\
\text { (Studs) }\end{array}$ & $\begin{array}{l}\text { Management } \\
\text { practices before } \\
\text { mating }\end{array}$ & $\begin{array}{c}\text { Management } \\
\text { practices during } \\
\text { pregnancy }\end{array}$ & $\begin{array}{c}\text { Management } \\
\text { practices during } \\
\text { kidding } \\
\end{array}$ & $\begin{array}{c}\text { Management } \\
\text { practices during } \\
\text { lactation } \\
\end{array}$ & $\begin{array}{l}\text { Young does up } \\
\text { to } 18 \text { months of } \\
\text { age }\end{array}$ \\
\hline $\begin{array}{l}1 \\
(1,7,8)\end{array}$ & $\begin{array}{l}\text { Does on veld, no } \\
\text { supplementation }\end{array}$ & $\begin{array}{l}\text { Does on veld, no } \\
\text { supplementation }\end{array}$ & $\begin{array}{l}\text { Does kid in veld, } \\
\text { no } \\
\text { supplementation }\end{array}$ & $\begin{array}{l}\text { Does on veld, } \\
\text { some years } \\
\text { supplemented with } \\
\text { chocolate grain }\end{array}$ & $\begin{array}{l}\text { Kids in veld - no } \\
\text { supplementation }\end{array}$ \\
\hline $\begin{array}{l}2 \\
(2,3,4,6,10)\end{array}$ & $\begin{array}{l}\text { Does on veld, no } \\
\text { supplementation }\end{array}$ & $\begin{array}{l}\text { Does on veld, no } \\
\text { supplementation }\end{array}$ & $\begin{array}{l}\text { Does kid on } \\
\text { pastures }\end{array}$ & $\begin{array}{l}\text { Does on veld, } \\
\text { some years } \\
\text { supplemented with } \\
\text { chocolate grain }\end{array}$ & $\begin{array}{l}\text { Kids in veld - no } \\
\text { supplementation }\end{array}$ \\
\hline $\begin{array}{l}3 \\
(5)\end{array}$ & $\begin{array}{l}\text { Does on veld, no } \\
\text { supplementation }\end{array}$ & $\begin{array}{l}\text { Does } \\
\text { supplemented with } \\
\text { energy blocks last } \\
\text { part of pregnancy }\end{array}$ & $\begin{array}{l}\text { Does kid on } \\
\text { pastures }\end{array}$ & $\begin{array}{l}\text { Does on veld, } \\
\text { some years } \\
\text { supplemented with } \\
\text { pellets / blocks }\end{array}$ & $\begin{array}{l}\text { Kids in veld - no } \\
\text { supplementation }\end{array}$ \\
\hline $\begin{array}{l}4 \\
(9,11)\end{array}$ & Flushing of does & $\begin{array}{l}\text { Does } \\
\text { supplemented with } \\
\text { energy licks last } \\
\text { part of pregnancy }\end{array}$ & $\begin{array}{l}\text { Does kid on } \\
\text { pastures }\end{array}$ & $\begin{array}{l}\text { Does on veld, } \\
\text { some years } \\
\text { supplemented with } \\
\text { licks }\end{array}$ & $\begin{array}{l}\text { Kids in veld - no } \\
\text { supplementation }\end{array}$ \\
\hline $\begin{array}{l}5 \\
(12)\end{array}$ & Does on pastures & Does on pastures & $\begin{array}{l}\text { Does kid on } \\
\text { pastures }\end{array}$ & Does on pastures & $\begin{array}{l}\text { All kids on } \\
\text { pastures }\end{array}$ \\
\hline
\end{tabular}

From the reproduction data the following reproduction parameters were calculated for each stud for each kidding season: number of kids scanned per 100 does scanned, number of does aborted per 100 does mated, number of does kidded per 100 does mated, number of kids born per 100 does mated, number of kids born per 100 does that kidded, percentage of stillborn kids, kid survival rate and number of kids weaned per 100 does mated. The average reproductive performance of does in each stud, in terms of these parameters, was calculated.

For the purpose of quantifying the effect of age, body weight and management system on reproductive performance, individual records collected from a specific doe over consecutive years were treated as uncorrelated. Least square means for reproductive traits were obtained by fitting the fixed effects year, stud, age of the doe and management system in a GLM-model (SAS, 2004), using all 14644 data records. The same model was used to determine the effect of management system during specific periods of the reproductive cycle on reproductive performance. Separate runs were done for management system before mating and management system during pregnancy.

As data recording started with the September 2000 kidding season, no body weights of the does before mating and at scanning were available for 2000. Therefore, only 10803 records of does with both body weight records were included in the analyses involving body weight. The GLM-model fitted to obtain least square means for body weight included the fixed effects of year, stud, age of the doe and management system (SAS, 2004). 
The data set also included data on body weights of kids. The effect of age of doe and doe management system on birth, weaning and 8-month body weight of kids was determined using 7432 records. The fixed effects included in the GLM-model were stud-year of birth, sex, birth/rearing status of the kid, age of dam, doe management system, all significant two-factor interactions and a covariate for age of the kid at weighing.

The 10803 records of does with both body weights before mating and at scanning were used to determine the effect of body weight on reproductive performance. The GLM-model fitted to obtain least square means for body weight included the fixed effects of year, stud, age of the doe, management system and reproductive status, viz. gave birth to normal kid(s), aborted, stillborn kid(s), kid(s) died after birth, doe did not kid. A linear regression of body weight on number of kids scanned, born and weaned was done, including fixed effects for year, stud, age of the doe and management system.

The effect of management system on body weight and reproductive performance at first mating of maiden does was evaluated using a GLM-model, which included fixed effects for stud and management system, fitted to 2099 maiden doe records.

The effect of body weight at first mating of maiden does on their first kidding performance was evaluated by dividing the body weight of these maiden does into six subclasses. The fixed effects for year, stud, body weight subclass and all significant two-factor interactions were included in the model fitted to 2099 maiden doe records to obtain least square means for number of kids scanned, born and weaned as well as kid survival rate at first kidding performance per body weight subclass.

\section{Results and Discussion}

The age structure of the does mated in the different flocks from 2000 to 2004 is presented in Table 2. It is evident that $10.7 \%$ of the does in the project were eight years and older.

Table 2 Age structure of the does in the data set

\begin{tabular}{|c|c|c|c|c|}
\hline \multirow[b]{2}{*}{ Doe age } & \multicolumn{2}{|c|}{ Does in full data set } & \multicolumn{2}{|c|}{ Does with mating and scanning weights } \\
\hline & $\begin{array}{c}\text { Number } \\
(\mathrm{n}=14644)\end{array}$ & $\%$ & $\begin{array}{c}\text { Number } \\
(\mathrm{n}=10803)\end{array}$ & $\%$ \\
\hline 2 & 2921 & 19.9 & 1914 & 17.7 \\
\hline 3 & 2855 & 19.5 & 2124 & 19.7 \\
\hline 4 & 2337 & 16.0 & 1773 & 16.4 \\
\hline 5 & 2054 & 14.0 & 1559 & 14.4 \\
\hline 6 & 1633 & 11.2 & 1203 & 11.1 \\
\hline 7 & 1273 & 8.7 & 985 & 9.1 \\
\hline 8 & 929 & 6.3 & 743 & 6.9 \\
\hline 9 & 447 & 3.1 & 373 & 3.5 \\
\hline 10 & 136 & 0.9 & 97 & 0.9 \\
\hline 11 & 46 & 0.3 & 26 & 0.2 \\
\hline 12 & 13 & 0.1 & 6 & 0.1 \\
\hline
\end{tabular}

The mean reproductive performance of the does in the different flocks from 2000 to 2004 is summarised in Table 3. Simple means are given to indicate the scope of variation among flocks. There was a wide range pertaining to reproductive performance among the different studs. Percentage of does scanned pregnant ranged from $75.8 \%$ to $92.3 \%$ among the different studs (average $=86.1 \%$ ), while scanning percentage ranged from $77.9 \%$ to $121.2 \%$ kids per doe scanned (average $=101.1 \%$ ). Kidding percentage (does kidded per 100 does mated) varied from $62.2 \%$ to $88.1 \%$ for the different studs (average $=79.8 \%$ ). 
Percentage multiple births varied between $0.6 \%$ and $37.9 \%$ (average $=19.3 \%$ ), while kid survival rate ranged from $80.6 \%$ to $92.2 \%$ (average $=85.6 \%$ ). Weaning percentage (kids weaned per 100 does mated) ranged from $54.4 \%$ to $105.3 \%$ (average $=80.6 \%$ ). Apart from a high kid mortality rate, which is considered by breeders as the most important factor contributing to low weaning percentages, $20 \%$ of the does did not conceive or lost their foetuses before birth. An average of $10.1 \%$ of the does that were scanned pregnant, did not kid. This implies that they have lost their kids between scanning and kidding.

Table 3 Mean reproductive performance of does in the different studs from 2000 to 2004

\begin{tabular}{|c|c|c|c|c|c|c|c|c|c|c|}
\hline Stud & $\begin{array}{c}\text { Does } \\
\text { scanned } \\
\text { pregnant / } \\
100 \text { does } \\
\text { scanned } \\
\end{array}$ & $\begin{array}{c}\text { Does } \\
\text { scanned } \\
\text { pregnant but } \\
\text { did not kid }\end{array}$ & $\begin{array}{c}\text { Kids } \\
\text { scanned / } \\
100 \text { does } \\
\text { scanned }\end{array}$ & $\begin{array}{c}\text { Does } \\
\text { kidded / } \\
100 \text { does } \\
\text { mated }\end{array}$ & $\begin{array}{c}\text { Does } \\
\text { aborted / } \\
100 \text { does } \\
\text { mated }\end{array}$ & $\begin{array}{c}\text { Kids born } \\
100 \text { does } \\
\text { kidded }\end{array}$ & $\begin{array}{c}\text { / Kids born / } \\
100 \text { does } \\
\text { mated }\end{array}$ & $\begin{array}{l}\text { Still- } \\
\text { born } \\
\text { kids } \\
(\%)\end{array}$ & $\begin{array}{c}\text { Kid } \\
\text { survival } \\
\text { rate (\%) }\end{array}$ & $\begin{array}{c}\text { Kids } \\
\text { weaned / } \\
100 \text { does } \\
\text { mated }\end{array}$ \\
\hline 1 & 91.2 & 6.7 & 104.3 & 85.4 & 5.6 & 118.2 & 101.1 & 2.1 & 92.2 & 91.4 \\
\hline 2 & 86.9 & 5.3 & 103.4 & 84.5 & 2.6 & 133.1 & 112.4 & 2.2 & 83.0 & 91.4 \\
\hline 3 & 84.7 & 18.8 & 86.6 & 72.4 & 2.8 & 106.2 & 76.9 & 1.4 & 86.4 & 65.6 \\
\hline 4 & 88.6 & 16.3 & 103.8 & 76.7 & 1.9 & 117.4 & 90.1 & 0.7 & 90.6 & 81.0 \\
\hline 5 & 83.0 & 6.49 & 104.3 & 82.7 & 4.2 & 119.2 & 98.5 & 2.2 & 80.6 & 77.5 \\
\hline 6 & 92.3 & 4.3 & 106.5 & 86.7 & 0.9 & 118.6 & 102.7 & 0.1 & 87.7 & 89.9 \\
\hline 7 & 76.3 & 19.5 & 79.7 & 68.1 & 10.0 & 105.7 & 72.0 & 2.1 & 82.0 & 57.8 \\
\hline 8 & 89.4 & 13.9 & 102.8 & 78.5 & 6.6 & 117.7 & 92.5 & 2.6 & 84.0 & 75.7 \\
\hline 9 & & & & 73.5 & 0.1 & 120.5 & 87.5 & 0.3 & 91.0 & 79.5 \\
\hline 10 & 75.8 & 11.4 & 77.9 & 62.2 & 4.6 & 100.6 & 62.5 & 1.1 & 88.0 & 54.4 \\
\hline 11 & 90.4 & 4.6 & 99.8 & 84.4 & 0.3 & 124.5 & 105.5 & 1.0 & 88.7 & 92.2 \\
\hline 12 & 88.5 & 3.5 & 121.2 & 88.1 & 0.1 & 137.9 & 121.5 & 1.1 & 87.6 & 105.3 \\
\hline Avg & 86.1 & 10.1 & 101.1 & 79.8 & 4.1 & 119.3 & 95.6 & 1.5 & 85.6 & 80.6 \\
\hline
\end{tabular}

Body weights of does before mating and at scanning 42 days after mating are given in Table 4 for the different studs. Body weight of does before mating ranged from $33.7 \mathrm{~kg}$ to $44.5 \mathrm{~kg}$ (average $=34.6 \mathrm{~kg}$ ) among studs, while body weight at scanning ranged from $37.0 \mathrm{~kg}$ to $46.4 \mathrm{~kg}$ (average $=38.6 \mathrm{~kg}$ ). Body weight of does differed significantly among all the studs.

The effect of doe age on reproductive performance and body weight of does is summarised in Table 5 . All reproductive parameters recorded had a typical inverted U-shaped relationship with age of dam, where the 2- and 3-year-old does and does older than 9 years of age reproduced worse $(P<0.01)$ than 4 - to 9-yearold does. Although 11- and 12-year-old does had high scanning percentages, their kidding percentages were considerably lower, indicating that they were more likely to lose their kids between scanning and kidding. The same trends were evident in all studs regardless of the reproductive rates of or management systems followed in the different studs. Body weight of does before mating increased from $26.8 \mathrm{~kg}$ in young does, to $37.7 \mathrm{~kg}$ in 5-year-old does, after which it increased steadily to $41.4 \mathrm{~kg}$ in 11-year-old does. Body weight of does at scanning followed the same trend.

Similar relationships to those of the reproduction traits were observed between age of dam and body weight of their kids (Figures 1 to 3). Kids born to young does (2-year-old does) had lower body weights up to 16 months of age than kids born to 4- to 8-year-old does. Birth, weaning and 8-month body weights of kids born to older does were also lower. 
Table 4 Body weight ( \pm s.e.) of does in the different studs

\begin{tabular}{ccc}
\hline Stud & Body weight before mating $(\mathrm{kg})$ & Body weight at scanning $(\mathrm{kg})$ \\
\hline 1 & $35.3 \pm 0.2$ & $42.4 \pm 0.3$ \\
2 & $36.3 \pm 0.2$ & $40.2 \pm 0.2$ \\
3 & $34.3 \pm 0.2$ & $37.3 \pm 0.2$ \\
4 & $37.9 \pm 0.3$ & $40.3 \pm 0.3$ \\
5 & $37.0 \pm 0.2$ & $37.3 \pm 0.2$ \\
6 & $39.8 \pm 0.3$ & $45.3 \pm 0.3$ \\
7 & $33.7 \pm 0.2$ & $39.1 \pm 0.3$ \\
8 & $37.6 \pm 0.2$ & $42.6 \pm 0.3$ \\
9 & & $37.0 \pm 0.2$ \\
10 & $41.1 \pm 0.4$ & $38.6 \pm 0.4$ \\
11 & $37.1 \pm 0.2$ & $43.7 \pm 0.2$ \\
12 & $44.5 \pm 0.2$ & $46.4 \pm 0.3$ \\
Average & $34.6 \pm 0.2$ & $38.6 \pm 0.2$ \\
\hline
\end{tabular}

Table 5 Effect of doe age on reproductive performance and body weight ( \pm s.e.) of does

\begin{tabular}{ccccccc}
\hline $\begin{array}{c}\text { Doe } \\
\text { age }\end{array}$ & $\begin{array}{c}\text { Number of kids } \\
\text { scanned per } \\
\text { doe scanned }\end{array}$ & $\begin{array}{c}\text { Number of } \\
\text { kids born per } \\
\text { doe mated }\end{array}$ & $\begin{array}{c}\text { Number of } \\
\text { kids weaned } \\
\text { per doe mated }\end{array}$ & $\begin{array}{c}\text { Number of } \\
\text { kids weaned } \\
\text { per kids born } \\
\text { alive }\end{array}$ & $\begin{array}{c}\text { Body weight } \\
\text { before mating } \\
\text { (kg) }\end{array}$ & $\begin{array}{c}\text { Body weight at } \\
\text { scanning (kg) }\end{array}$ \\
\hline 2 & $0.78 \pm 0.01$ & $0.70 \pm 0.01$ & $0.56 \pm 0.01$ & $0.81 \pm 0.01$ & $26.8 \pm 0.1$ & $30.7 \pm 0.1$ \\
3 & $0.94 \pm 0.01$ & $0.89 \pm 0.01$ & $0.76 \pm 0.01$ & $0.86 \pm 0.01$ & $32.3 \pm 0.1$ & $36.6 \pm 0.1$ \\
4 & $1.07 \pm 0.01$ & $1.05 \pm 0.01$ & $0.90 \pm 0.01$ & $0.88 \pm 0.01$ & $35.7 \pm 0.1$ & $40.3 \pm 0.1$ \\
5 & $1.12 \pm 0.01$ & $1.09 \pm 0.01$ & $0.95 \pm 0.01$ & $0.90 \pm 0.01$ & $37.7 \pm 0.1$ & $42.4 \pm 0.1$ \\
6 & $1.16 \pm 0.01$ & $1.12 \pm 0.02$ & $0.95 \pm 0.02$ & $0.90 \pm 0.01$ & $38.7 \pm 0.1$ & $43.5 \pm 0.1$ \\
7 & $1.19 \pm 0.02$ & $1.17 \pm 0.02$ & $0.99 \pm 0.02$ & $0.91 \pm 0.01$ & $39.3 \pm 0.1$ & $44.0 \pm 0.2$ \\
8 & $1.13 \pm 0.02$ & $1.07 \pm 0.02$ & $0.91 \pm 0.02$ & $0.89 \pm 0.01$ & $39.4 \pm 0.2$ & $43.7 \pm 0.2$ \\
9 & $1.11 \pm 0.03$ & $1.04 \pm 0.03$ & $0.84 \pm 0.03$ & $0.86 \pm 0.02$ & $39.6 \pm 0.2$ & $43.4 \pm 0.2$ \\
10 & $0.99 \pm 0.05$ & $0.84 \pm 0.06$ & $0.69 \pm 0.06$ & $0.87 \pm 0.04$ & $40.5 \pm 0.4$ & $44.2 \pm 0.5$ \\
11 & $1.09 \pm 0.09$ & $0.84 \pm 0.10$ & $0.68 \pm 0.11$ & $0.95 \pm 0.08$ & $41.4 \pm 0.8$ & $44.8 \pm 0.9$ \\
12 & $1.10 \pm 0.19$ & $0.81 \pm 0.21$ & $0.80 \pm 0.22$ & $0.96 \pm 0.15$ & $39.6 \pm 1.7$ & $42.5 \pm 1.9$ \\
\hline
\end{tabular}

The effect of management system on reproductive performance is presented in Table 6 for the five major systems followed in the different studs. Management system had a significant $(\mathrm{P}<0.01)$ influence on reproductive performance of does in the different studs. Higher reproductive rates were observed under those management systems involving additional or supplementary feeding at various stages of the reproductive cycle. 


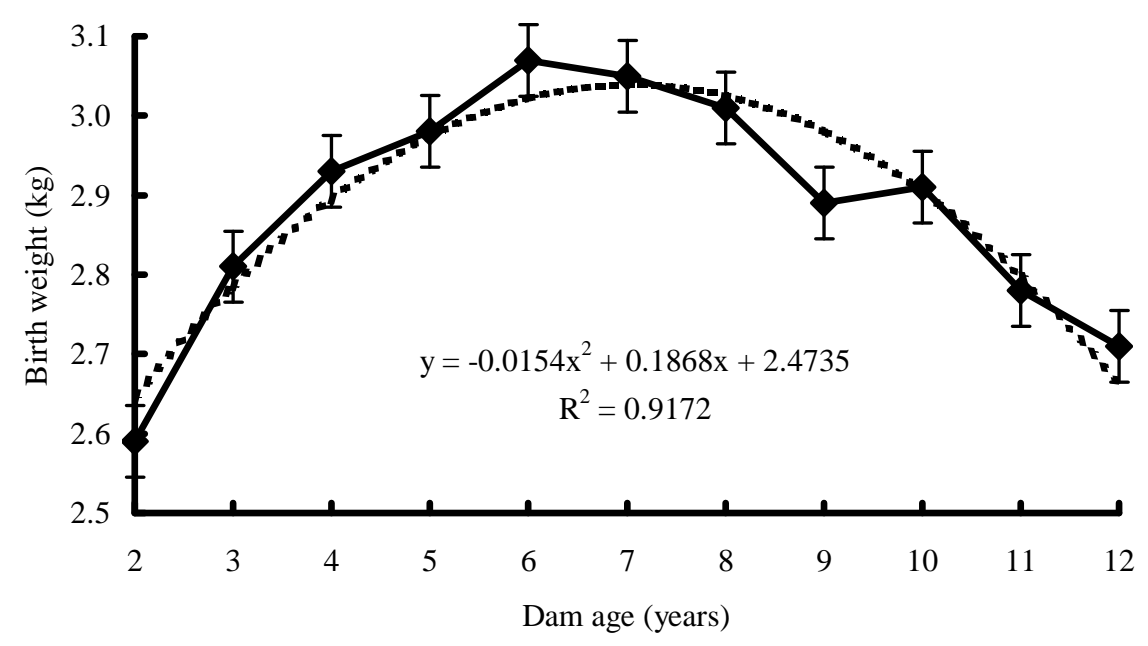

Figure 1 Relationship between dam age and birth weight of kids.

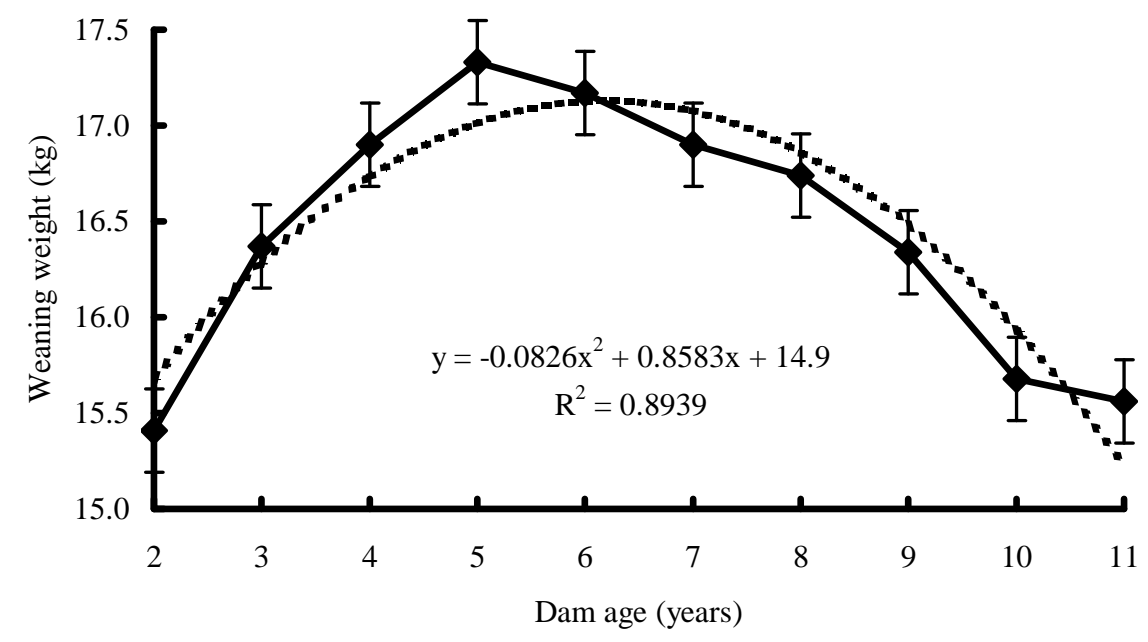

Figure 2 Relationship between dam age and weaning weight of kids.

The effect of management system during specific periods of the reproductive cycle on reproductive performance is presented in Tables 7 and 8 . From Table 7 it is evident that does that received either flushing treatment or were run on pastures before mating, performed better than does that were run on veld without any supplementation before mating. There was no difference in the percentage of does scanned pregnant between those does that were supplemented and those does that were run on veld without supplementation during pregnancy (Table 8). However, fewer of the supplemented does aborted and more kidded than the does not supplemented. 


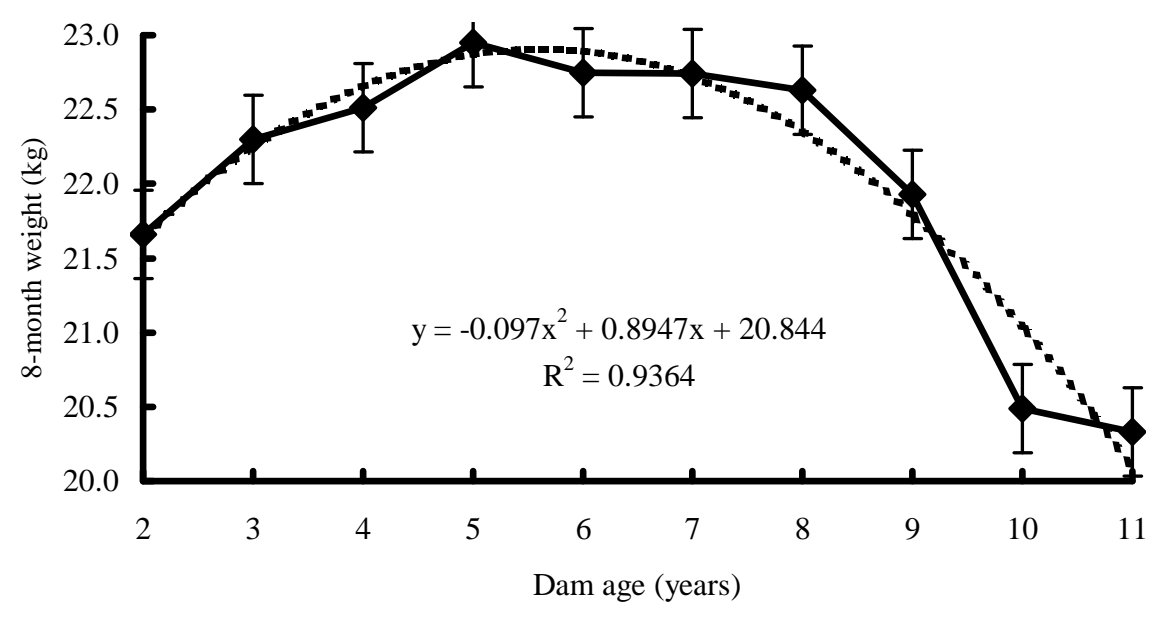

Figure 3 Relationship between doe age and 8-month body weight of kids.

Table 6 Effect of management system on reproductive performance of does

\begin{tabular}{|c|c|c|c|c|c|}
\hline \multirow{2}{*}{ Trait } & \multicolumn{5}{|c|}{ Management system } \\
\hline & 1 & 2 & 3 & 4 & 5 \\
\hline $\begin{array}{l}\text { Does scanned pregnant } \\
\text { / } 100 \text { does scanned }\end{array}$ & $0.85^{\mathrm{ab}} \pm 0.02$ & $0.87^{\mathrm{c}} \pm 0.01$ & $0.83^{\text {cde }} \pm 0.02$ & $0.90^{\text {ad }} \pm 0.02$ & $0.89^{\text {be }} \pm 0.02$ \\
\hline $\begin{array}{l}\text { Does scanned pregnant } \\
\text { and kidded }\end{array}$ & $0.82^{\mathrm{ab}} \pm 0.01$ & $0.84^{\mathrm{cd}} \pm 0.01$ & $0.89^{\mathrm{ac}} \pm 0.01$ & $0.92^{\text {bd }} \pm 0.02$ & $0.93^{\mathrm{ac}} \pm 0.02$ \\
\hline $\begin{array}{l}\text { Kids scanned / } 100 \\
\text { does scanned }\end{array}$ & $0.98^{\mathrm{ab}} \pm 0.03$ & $1.03^{\mathrm{b}} \pm 0.03$ & $1.06^{\mathrm{a}} \pm 0.03$ & $1.12^{\mathrm{a}} \pm 0.03$ & $1.25^{\mathrm{a}} \pm 0.03$ \\
\hline $\begin{array}{l}\text { Does kidded / } 100 \text { does } \\
\text { mated }\end{array}$ & $0.73^{\mathrm{ab}} \pm 0.02$ & $0.78^{\mathrm{ab}} \pm 0.02$ & $0.80^{\mathrm{ab}} \pm 0.02$ & $0.86^{\mathrm{a}} \pm 0.02$ & $0.87^{\mathrm{b}} \pm 0.02$ \\
\hline $\begin{array}{l}\text { Kids born / } 100 \text { does } \\
\text { mated }\end{array}$ & $0.81^{\mathrm{ab}} \pm 0.03$ & $0.93^{\mathrm{a}} \pm 0.03$ & $0.93^{b} \pm 0.03$ & $1.07^{\mathrm{a}} \pm 0.03$ & $1.20^{\mathrm{a}} \pm 0.03$ \\
\hline $\begin{array}{l}\text { Kids born / } 100 \text { does } \\
\text { kidded }\end{array}$ & $1.12^{\mathrm{a}} \pm 0.02$ & $1.19^{\mathrm{a}} \pm 0.02$ & $1.16^{\mathrm{a}} \pm 0.02$ & $1.23^{\mathrm{a}} \pm 0.02$ & $1.37^{\mathrm{a}} \pm 0.02$ \\
\hline $\begin{array}{l}\text { Kids weaned / } 100 \\
\text { does mated }\end{array}$ & $0.71^{a} \pm 0.03$ & $0.79^{\mathrm{ab}} \pm 0.03$ & $0.73^{b} \pm 0.03$ & $0.91^{\mathrm{ab}} \pm 0.03$ & $1.06^{\mathrm{ab}} \pm 0.03$ \\
\hline
\end{tabular}

abcde Means with the same superscripts differ significantly $(\mathrm{P}<0.01)$ within a specific trait.

The effect of body weight before mating and at scanning on reproductive performance is summarised in Table 10. Body weights before mating and at scanning had a significant positive relationship with number of kids born per doe mated, number of multiple births and number of kids weaned per doe mated. Does carrying twins had higher body weights at scanning than dry does or does carrying single kids. For every kilogram increase in body weight before mating, 0.0237 and 0.0218 more kids were born and weaned respectively.

The effect of management system on body weights of does and kids is summarised in Table 9 . Management system also had a significant influence on body weight of does and kids. 
Table 7 Effect of management system before mating on reproductive performance of does

\begin{tabular}{lccc}
\hline \multirow{2}{*}{ Trait } & \multicolumn{3}{c}{ Management system before mating } \\
\cline { 2 - 4 } & Flushing of does & Does on pastures & Does on veld, no supplementation \\
\hline $\begin{array}{l}\text { Does scanned pregnant / } \\
\begin{array}{l}\text { 100 does scanned } \\
\text { Does scanned pregnant } \\
\text { and kidded }\end{array}\end{array}$ & $0.90^{\mathrm{a}} \pm 0.02$ & $0.89^{\mathrm{b}} \pm 0.02$ & $0.85^{\mathrm{ab}} \pm 0.01$ \\
$\begin{array}{l}\text { Kids scanned / } 100 \text { does } \\
\text { scanned }\end{array}$ & $0.92^{\mathrm{a}} \pm 0.02$ & $0.93^{\mathrm{b}} \pm 0.02$ & $0.85^{\mathrm{ab}} \pm 0.01$ \\
$\begin{array}{l}\text { Does kidded / } 100 \text { does } \\
\text { mated }\end{array}$ & $0.86^{\mathrm{a}} \pm 0.02$ & $1.22^{\mathrm{a}} \pm 0.03$ & $1.02^{\mathrm{a}} \pm 0.03$ \\
$\begin{array}{l}\text { Kids born / } 100 \text { does } \\
\text { mated }\end{array}$ & $1.07^{\mathrm{a}} \pm 0.03$ & $0.88^{\mathrm{b}} \pm 0.02$ & $0.77^{\mathrm{ab}} \pm 0.01$ \\
$\begin{array}{l}\text { Kids born / } 100 \text { does } \\
\text { kidded }\end{array}$ & $1.23^{\mathrm{a}} \pm 0.02$ & $1.21^{\mathrm{a}} \pm 0.03$ & $0.91^{\mathrm{a}} \pm 0.03$ \\
$\begin{array}{l}\text { Kids weaned / } 100 \text { does } \\
\text { mated }\end{array}$ & $0.91^{\mathrm{a}} \pm 0.03$ & $1.38^{\mathrm{a}} \pm 0.02$ & $1.17^{\mathrm{a}} \pm 0.02$ \\
\end{tabular}

${ }^{\mathrm{ab}}$ Means with the same superscripts differ significantly $(\mathrm{P}<0.01)$ within a specific trait.

Table 8 Effect of management system during pregnancy on reproductive performance of does

\begin{tabular}{|c|c|c|c|}
\hline \multirow[b]{2}{*}{ Trait } & \multicolumn{3}{|c|}{ Management system during pregnancy } \\
\hline & $\begin{array}{l}\text { Does supplemented with energy } \\
\text { blocks last part of pregnancy }\end{array}$ & Does on pastures & $\begin{array}{l}\text { Does on veld, no } \\
\text { supplementation }\end{array}$ \\
\hline $\begin{array}{l}\text { Does scanned pregnant / } \\
100 \text { does scanned }\end{array}$ & $0.86^{\mathrm{a}} \pm 0.01$ & $0.89^{\mathrm{ab}} \pm 0.02$ & $0.86^{b} \pm 0.01$ \\
\hline $\begin{array}{l}\text { Does scanned pregnant } \\
\text { and kidded }\end{array}$ & $0.92^{\mathrm{a}} \pm 0.01$ & $0.93^{b} \pm 0.02$ & $0.84^{\mathrm{ab}} \pm 0.01$ \\
\hline $\begin{array}{l}\text { Kids scanned / } 100 \text { does } \\
\text { scanned }\end{array}$ & $1.09^{\mathrm{a}} \pm 0.03$ & $1.26^{\mathrm{a}} \pm 0.03$ & $1.01^{\mathrm{a}} \pm 0.03$ \\
\hline $\begin{array}{l}\text { Does kidded / } 100 \text { does } \\
\text { mated }\end{array}$ & $0.83^{a} \pm 0.02$ & $0.88^{\mathrm{a}} \pm 0.02$ & $0.76^{\mathrm{a}} \pm 0.02$ \\
\hline $\begin{array}{l}\text { Kids born / } 100 \text { does } \\
\text { mated }\end{array}$ & $0.99^{\mathrm{a}} \pm 0.03$ & $1.21^{\mathrm{a}} \pm 0.03$ & $0.89^{a} \pm 0.03$ \\
\hline $\begin{array}{l}\text { Kids born / } 100 \text { does } \\
\text { kidded }\end{array}$ & $1.19^{\mathrm{a}} \pm 0.02$ & $1.38^{\mathrm{ab}} \pm 0.02$ & $1.17^{\mathrm{b}} \pm 0.02$ \\
\hline $\begin{array}{l}\text { Kids weaned / } 100 \text { does } \\
\text { mated }\end{array}$ & $0.81^{a} \pm 0.03$ & $1.06^{\mathrm{a}} \pm 0.03$ & $0.76^{\mathrm{a}} \pm 0.03$ \\
\hline
\end{tabular}

The effect of management system on body weight and reproduction of maiden does is presented in Table 11. Body weight before mating of young does run on pastures until 18 months of age, was higher than that of maiden does in the other management systems, with resultant higher reproductive rates. The effect of body weight at first mating on the reproductive performance of these maiden does is summarised in Table 12. Number of kids scanned, born and weaned per maiden doe at first kidding increased with an increase in body weight at first mating. 
Table 9 Effect of doe management system on body weight ( \pm s.e.) of does and kids

\begin{tabular}{cccccc}
\hline $\begin{array}{c}\text { Management } \\
\text { system }\end{array}$ & $\begin{array}{c}\text { Body weight of } \\
\text { does before } \\
\text { mating }(\mathrm{kg})\end{array}$ & $\begin{array}{c}\text { Body weight of } \\
\text { does at scanning } \\
(\mathrm{kg})\end{array}$ & $\begin{array}{c}\text { Birth weight of } \\
\text { kids }(\mathrm{kg})\end{array}$ & $\begin{array}{c}\text { Weaning weight } \\
\text { of kids }(\mathrm{kg})\end{array}$ & $\begin{array}{c}\text { 8-month body } \\
\text { weight of kids } \\
(\mathrm{kg})\end{array}$ \\
\hline 1 & $35.3^{\mathrm{abc}} \pm 0.2$ & $40.9^{\mathrm{a}} \pm 0.2$ & $2.88^{\mathrm{a}} \pm 0.04$ & $15.4^{\mathrm{b}} \pm 0.37$ & $19.7^{\mathrm{a}} \pm 0.53$ \\
2 & $36.1^{\mathrm{abc}} \pm 0.2$ & $39.5^{\mathrm{a}} \pm 0.2$ & $3.21^{\mathrm{a}} \pm 0.04$ & $16.9^{\mathrm{abc}} \pm 0.37$ & $21.5^{\mathrm{a}} \pm 0.52$ \\
3 & $36.7^{\mathrm{b}} \pm 0.2$ & $36.9^{\mathrm{a}} \pm 0.2$ & $3.06^{\mathrm{a}} \pm 0.04$ & $14.7^{\mathrm{abc}} \pm 0.37$ & $20.7^{\mathrm{a}} \pm 0.52$ \\
4 & $36.8^{\mathrm{c}} \pm 0.2$ & $43.3^{\mathrm{a}} \pm 0.2$ & $2.72^{\mathrm{a}} \pm 0.04$ & $15.7^{\mathrm{c}} \pm 0.40$ & $22.4^{\mathrm{a}} \pm 0.55$ \\
5 & $44.2^{\mathrm{abc}} \pm 0.2$ & $45.9^{\mathrm{a}} \pm 0.3$ & $2.55^{\mathrm{a}} \pm 0.04$ & $20.5^{\mathrm{abc}} \pm 0.40$ & $26.5^{\mathrm{a}} \pm 0.56$ \\
\hline
\end{tabular}

${ }^{\mathrm{ab}}$ Means with the same superscripts differ significantly $(\mathrm{P}<0.01)$ within a specific trait.

Table 10 Effect of body weight ( \pm s.e.) on reproductive performance of does

\begin{tabular}{|c|c|c|}
\hline Trait & Body weight before mating (kg) & Body weight at scanning (kg) \\
\hline \multicolumn{3}{|l|}{ Reproductive status } \\
\hline $\begin{array}{l}\text { 1. Gave birth to normal kid/s } \\
\text { 2. Aborted } \\
\text { 3. Stillborn kid/s } \\
\text { 4. Kid/s died after birth } \\
\text { 5. Doe did not kid }\end{array}$ & $\begin{array}{l}37.6^{\mathrm{a}} \pm 0.2 \\
37.4^{\mathrm{b}} \pm 0.3 \\
37.4^{\mathrm{c}} \pm 0.4 \\
38.0^{\mathrm{d}} \pm 0.4 \\
36.2^{\mathrm{abcd}} \pm 0.2 \\
\end{array}$ & $\begin{array}{l}41.9^{\mathrm{abc}} \pm 0.2 \\
40.9^{\mathrm{a}} \pm 0.3 \\
41.1^{\mathrm{b}} \pm 0.5 \\
40.9^{\mathrm{c}} \pm 0.4 \\
39.3^{\mathrm{abc}} \pm 0.2\end{array}$ \\
\hline $\begin{array}{l}\text { Number of kids scanned per d } \\
0 \\
1 \\
2 \\
3 \\
\text { Regression } \\
\end{array}$ & $\begin{array}{l}35.9^{\mathrm{a}} \pm 0.2 \\
37.1^{\mathrm{a}} \pm 0.2 \\
39.4^{\mathrm{a}} \pm 0.2 \\
38.5 \pm 1.6 \\
\mathrm{y}=0.1947+0.0237 \mathrm{x}\end{array}$ & $\begin{array}{l}38.7^{\mathrm{a}} \pm 0.2 \\
41.2^{\mathrm{a}} \pm 0.2 \\
44.4^{\mathrm{a}} \pm 0.2 \\
42.6 \pm 1.7 \\
\mathrm{y}=-0.2272+0.0316 \mathrm{x}\end{array}$ \\
\hline $\begin{array}{l}\text { Number of kids born per doe r } \\
0 \\
1 \\
2 \\
3 \\
\text { Regression } \\
\end{array}$ & $\begin{array}{l}36.4^{\mathrm{abc}} \pm 0.2 \\
37.2^{\mathrm{abc}} \pm 0.2 \\
39.2^{\mathrm{b}} \pm 0.2 \\
40.3^{\mathrm{c}} \pm 0.7 \\
\mathrm{y}=0.0397+0.0237 \mathrm{x}\end{array}$ & $\begin{array}{l}39.5^{\mathrm{a}} \pm 0.2 \\
41.4^{\mathrm{a}} \pm 0.2 \\
44.1^{\mathrm{a}} \pm 0.2 \\
46.4^{\mathrm{a}} \pm 0.7 \\
\mathrm{y}=-0.4684+0.0334 \mathrm{x}\end{array}$ \\
\hline $\begin{array}{l}\text { Number of kids weaned per do } \\
0 \\
1 \\
2 \\
3 \\
\text { Regression }\end{array}$ & $\begin{array}{l}\text { ated } \\
36.6^{\mathrm{abc}} \pm 0.2 \\
37.3^{\mathrm{abc}} \pm 0.2 \\
39.3^{\mathrm{b}} \pm 0.2 \\
41.2^{\mathrm{c}} \pm 1.1 \\
\mathrm{y}=0.0781+0.0218 \mathrm{x}\end{array}$ & $\begin{array}{l}40.1^{\mathrm{abc}} \pm 0.2 \\
41.6^{\mathrm{abc}} \pm 0.2 \\
44.2^{\mathrm{b}} \pm 0.2 \\
45.6^{\mathrm{c}} \pm 1.2 \\
\mathrm{y}=-0.3484+0.0299 \mathrm{x}\end{array}$ \\
\hline
\end{tabular}

abcd Means with the same superscripts differ significantly $(\mathrm{P}<0.01)$ within a specific trait.

\section{Discussion}

From the results it is evident that reproduction of Angora does depends on body weight, age and management system. The effect of body weight and flushing on ovulation rate and reproductive performance in sheep has been well documented (Cockrem, 1979; Gunn et al., 1984). More does in this study that received either flushing treatment or were run on pastures before mating were pregnant at scanning than does that were run on veld without any supplementation before mating. 
Table 11 Effect of management system on reproductive performance and body weight ( \pm s.e.) of maiden does

\begin{tabular}{|c|c|c|c|c|c|}
\hline $\begin{array}{c}\text { Management } \\
\text { system }\end{array}$ & $\begin{array}{l}\text { Number of kids } \\
\text { scanned per doe } \\
\text { scanned }\end{array}$ & $\begin{array}{c}\text { Number of kids } \\
\text { born per doe } \\
\text { mated }\end{array}$ & $\begin{array}{c}\text { Number of kids } \\
\text { weaned per doe } \\
\text { mated }\end{array}$ & $\begin{array}{l}\text { Body weight } \\
\text { before mating } \\
(\mathrm{kg})\end{array}$ & $\begin{array}{l}\text { Body weight at } \\
\text { scanning (kg) }\end{array}$ \\
\hline 1 & $0.71^{\mathrm{ab}} \pm 0.02$ & $0.60^{\mathrm{abcd}} \pm 0.03$ & $0.45^{\mathrm{a}} \pm 0.03$ & $25.2^{\mathrm{a}} \pm 0.2$ & $29.5^{\mathrm{a}} \pm 0.2$ \\
\hline 2 & $0.75^{\mathrm{cd}} \pm 0.02$ & $0.66^{\mathrm{acd}} \pm 0.02$ & $0.54^{\mathrm{a}} \pm 0.02$ & $25.7^{b} \pm 0.1$ & $28.8^{a} \pm 0.2$ \\
\hline 3 & $0.68^{\text {ef }} \pm 0.03$ & $0.72^{\text {bcd }} \pm 0.04$ & $0.51^{b} \pm 0.04$ & $25.8^{c} \pm 0.3$ & $29.0^{b} \pm 0.3$ \\
\hline 4 & $0.90^{\text {ace }} \pm 0.03$ & $0.89^{c} \pm 0.04$ & $0.72^{\mathrm{ab}} \pm 0.04$ & $26.9^{\mathrm{abc}} \pm 0.3$ & $33.2^{\mathrm{ab}} \pm 0.3$ \\
\hline 5 & $0.99^{\mathrm{bdf}} \pm 0.03$ & $0.99^{\mathrm{d}} \pm 0.03$ & $0.84^{\mathrm{ab}} \pm 0.03$ & $35.0^{\mathrm{abc}} \pm 0.3$ & $37.1^{\mathrm{ab}} \pm 0.3$ \\
\hline
\end{tabular}

abcd Means with the same superscripts differ significantly $(\mathrm{P}<0.01)$ within a specific trait.

Table 12 Effect of body weight ( \pm s.e.) at first mating on reproductive performance of maiden does

\begin{tabular}{cccccc}
\hline $\begin{array}{c}\text { Weight class } \\
(\mathrm{kg})\end{array}$ & $\mathrm{n}$ & $\begin{array}{c}\text { Number of kids } \\
\text { scanned per doe } \\
\text { scanned }\end{array}$ & $\begin{array}{c}\text { Number of kids } \\
\text { born per doe mated }\end{array}$ & $\begin{array}{c}\text { Number of kids } \\
\text { weaned per doe } \\
\text { mated }\end{array}$ & $\begin{array}{c}\text { Number of kids } \\
\text { weaned per kids } \\
\text { born alive }\end{array}$ \\
\hline $\begin{array}{l}1 .<20.0 \\
120\end{array}$ & $0.32^{23456} \pm 0.04$ & $0.29^{23456} \pm 0.05$ & $0.24^{23456} \pm 0.05$ & $0.82 \pm 0.09$ \\
2. $20.0-24.9$ & 717 & $0.64^{13456} \pm 0.02$ & $0.54^{13456} \pm 0.02$ & $0.41^{13456} \pm 0.02$ & $0.76 \pm 0.02$ \\
3. $25.0-29.9$ & 813 & $0.81^{126} \pm 0.02$ & $0.77^{1246} \pm 0.02$ & $0.62^{1246} \pm 0.02$ & $0.82 \pm 0.02$ \\
4. $30.0-34.9$ & 311 & $0.84^{126} \pm 0.03$ & $0.87^{123} \pm 0.03$ & $0.75^{123} \pm 0.03$ & $0.87 \pm 0.03$ \\
$5.35 .0-39.9$ & 104 & $0.94^{12} \pm 0.06$ & $0.88^{12} \pm 0.06$ & $0.77^{12} \pm 0.06$ & $0.86 \pm 0.05$ \\
$6 .>40.0$ & 34 & $1.09^{1234} \pm 0.09$ & $1.08^{123} \pm 0.09$ & $0.92^{123} \pm 0.10$ & $0.88 \pm 0.08$
\end{tabular}

123456 Specific trait differed significantly $(\mathrm{P}<0.05)$ from those weight classes indicated in the superscripts.

In this survey, $14 \%$ of the does did not carry a kid(s) at scanning. It is well established that $20-30 \%$ of sheep embryos die during the first weeks of pregnancy (Edey, 1969; Kelly, 1984). The factors implicated in these losses still have to be fully explained. It is also known that progesterone plays a crucial role in maintaining pregnancy in the doe (Denamur, 1974). Nutrition during early pregnancy and peripheral progesterone concentration are inversely related (Parr et al., 1982; Williams \& Cumming, 1982; Parr et al., 1987; McKelvey \& Robinson, 1988; Ashworth, 1995). Sheep fed high-energy rations after mating during the first part of gestation had reduced progesterone concentrations and showed an increase in embryo mortality. Low-plane feeding at this time had little effect on embryo survival or plasma progesterone concentration (Mani et al., 1995). However, embryos of young does and older does in poor condition at mating are at risk (Robinson, 1983). Most of the does in this survey were run on veld from mating through the first part of pregnancy. Overfeeding just after mating is therefore not a problem that would adversely affect embryo survival.

When undernutrition is severe, however, there can be a significant decrease is pregnancy rate in sheep, although this does not appear to be attributable to any inadequacy in corpus luteum function (Abecia et al., 1994). Nutritional restriction during mid-pregnancy may have a significant effect on foetal mortality (Kelly et al., 1989). Osuagwuh (1992) also concluded that for economical animal production it is not necessary to feed pregnant West African Dwarf goats on high concentrate rations throughout pregnancy, but they should not be underfed between days 61 and 120 of pregnancy. In the present survey, $84 \%$ of the does that were run on veld without supplementation during pregnancy and was scanned pregnant, did eventually kid, compared 
to 92 and $93 \%$ of the does that either received supplementation during the last part of pregnancy or were run on pastures.

Mellado et al. (2004) also emphasised the importance of removing risk factors such as low body energy reserves, low serum glucose levels, young does and low manganese and calcium intake at mating for improving the reproductive performance of goats under natural grazing conditions.

Louw (1981) reported that low energy nutrition of Angora goats during the third trimester of pregnancy could cause poor udder development and delay the onset of milk and colostrum production. Low protein and/or energy intake during this period could also lead to a poor mothering instinct. Most veld types in the Angora goat farming areas are unable to fulfil the protein and energy requirements of late pregnant and lactating Angora goat does (Louw, 1981; Wentzel, 1982). Although information on the feed requirements of these animals and the degree in which natural grazing supplies this, is relatively limited, there is at least sufficient proof that the most important deficiency experienced by does and growing animals is that of energy. It is also not surprising that the major problems in the Angora goat industry can be traced to an energy deficiency (Wentzel, 1982). Occasional examples of this deficiency are low conception rates, high rates of abortion, high peri-natal losses, poor milk production in lactating does, poor growth in small kids and young goats and high losses during unfavourable weather conditions. The elimination of these problems on farms through the supply of the necessary energy supplementation is sufficient proof that optimal production and reproduction in the Angora goat are possible in practice through the correction of a single deficiency (Wentzel, 1982). The general lower reproductive performance of does under Management system 1 (does run continuously under veld conditions), is further evidence that veld alone cannot adequately supply the nutritional requirements of pregnant and lactating Angora goat does.

Doe selection has a dual purpose. The first is the immediate improvement of the productive performance of the flock and, therefore, the income from the flock. The second aim is long-term genetic improvement. To optimise the rate of genetic improvement, a balance between selection intensity (number of replacement animals needed per year) and generation interval (the average age of the parents when the progeny is born) must be established. The most important factor that will determine replacement rate, and hence the number of doe age groups that can be kept, is kidding percentage and kid survival rate. The more young goats available at selection age, the more animals can be replaced. With a weaning percentage of $80 \%$, replacement rates of 20 to 25\% could easily be achieved. Analysis of data on South African stud Angora goats revealed a generation interval of 5.11 years, which could be reduced considerably. Theoretically, genetic progress in selection for a single trait could be increased by approximately $12 \%$ if the number of doe age groups is decreased to five and that of ram groups to two. This issue should be addressed in the Angora goat industry.

It could be argued that a high proportion of young does in the flock (with fewer doe age groups) will negatively affect the current performance of the flock due to the generally lower efficiency of young does. This should be weighed against achieving optimum genetic gain as well as the lower efficiency of older does.

The lower reproductive efficiency of young does could partly be ascribed to their lower body weight. A body weight of 25 to $27 \mathrm{~kg}$ at 18 months of age is generally regarded as a minimum to ensure that young does conceive and are able to carry their foetuses to parturition. However, $40 \%$ of young does in this study had mating body weights below $25 \mathrm{~kg}$; the range being from 15 to $45.8 \mathrm{~kg}$, depending on the respective rearing environments. Young does with mating weights below $25 \mathrm{~kg}$ also had lower survival rates of their kids.

From the results on post-weaning growth rates of kids in the same survey (Snyman, 2007), it was evident that the growth rate of doe kids is severely stunted after weaning, for at least an 8-month period. This contributes directly to the young does not being able to reach an acceptable weight before mating at 18 months of age. It would be advantageous to take special care that doe kids have every opportunity to grow out sufficiently to reach the required body weight before mating at 18 months of age. The high reproductive rates achieved by maiden does under Management system 5 are evidence that young does that had the opportunity to grow out sufficiently, are able to reproduce well. This is evident from the higher conception rate $(\mathrm{P}<0.01)$ of these young does. The better nutritional conditions that these does were under during pregnancy also contributed to lower losses between scanning and kidding. 


\section{Conclusions}

From the results presented, it is evident that age of the doe, body weight and management system all have a significant effect on reproduction of Angora goats. The practice of keeping does older than seven to eight years of age in the breeding flock is detrimental for optimising overall productivity. Not only will it slow down genetic progress by increasing generation interval, but income of the current herd is also adversely affected by retaining unproductive older does. To optimise productive and reproductive efficiency and rate of genetic progress, breeders should keep a maximum of 5 to 6 dam age groups.

Furthermore, it is imperative that breeders ensure that young replacement does have every opportunity to grow out sufficiently to reach the required body weight before mating at 18 months of age. Older does should also be in a good body condition before the onset of the reproductive cycle. The nutrition of does throughout the reproductive cycle should be adequate to ensure optimum reproduction, with emphasis on certain critical periods. These are before, during and directly after mating, to ensure successful ovulation, fertilization, morula formation, blastocyst implantation and embryonic development. The second important period is the third trimester of pregnancy. Adequate nutrition during this period will ensure good udder development and early onset of milk and colostrum production, as well as viable and healthy kids. Management during kidding and nutrition during the first six to eight weeks of lactation is also imperative to ensure a high weaning percentage.

The results obtained with this survey supplied a good basis for scientific research into specific aspects of the reproductive cycle of the Angora goat.

\section{Acknowledgements}

The author wishes to convey her sincere appreciation to all people who participated in the project and to Mohair South Africa for funding the project.

\section{References}

Abecia, J.A., Rhind, S.M. \& Mcmillen, S.R., 1994. Effect of undernutrition on luteal function and the distribution of progesterone in endometrial tissue in ewes. ITEA, Prod. Anim. 90A (2), 63-71.

Ashworth, C.J., 1995. Maternal and conceptus factors affecting histotrophic nutrition and survival of embryos. Livest. Prod. Sci. 44, 99-105.

Cockrem, F.R.M., 1979. A review of the influence of live weight and flushing on fertility made in the context of efficient sheep production. Proc. N. Z. Soc. Anim. Prod. 39, 23-42.

Constantinou, A., 1989. Genetic and environmental relationships of body weight, milk yield and litter size in Damascus goats. Small Rumin. Res. 2, 163-174.

Denamur, R., 1974. Luteotrophic factors in the sheep. J. Reprod. Fert. 38, 251.

Edey, T.N., 1969. Prenatal mortality in sheep: A review. Anim. Breed. Abstr. 37, 173-190.

Geyer, A.C., 1998. Strategiese beplanning en risikobestuur vir ekstensiewe Angorabokboerdery in die Karoo. Ph.D thesis, University of the Orange Free State, Bloemfontein.

Gunn, R.G., Doney, J.M. \& Smith, W.F., 1984. The effect of level of pre-mating nutrition on ovulation rate in Scottish Blackface ewes in different body conditions at mating. Anim. Prod. 39, 235-239.

Kelly, R.W., 1984. Fertilization failure and embryonic wastage. In: Reproduction in Sheep. Eds Lindsay, D.R. \& Pearce, D.T., Cambridge University Press, Cambridge. pp. 127-133.

Kelly, R.W., Wilkens, J.F. \& Newnham, J.P., 1989. Fetal mortality from day 30 of pregnancy in Merino ewes offered different levels of nutrition. Aust. J. Exp. Agric. 29, 339-342.

Louw, D.J., 1981. Invloed van energie- en proteïenvoeding op melkproduksie van Angorabokooie. Karoo Agric. 2 (2), 29-34.

Mani, A.U., Watson, E.D. \& Mckelvey, W.A.C., 1995. Effect of undernutrition on progesterone concentration during the early luteal phase and mid-gestation in goats. Vet. Rec. 136, 518-519.

Mckelvey, W.A. \& Robinson, J.J., 1988. The use of reciprocal embryo transfer to separate the effects of preand post-mating nutrition on embryo survival and growth of the ovine conceptus. Proc. $11^{\text {th }}$ Int. Cong. of Anim. Reprod. Artificial Insemination, (Dublin) 2, Paper No. 176.

Mellado, M., Valdez, R., Lara, L.M. \& Garcia, J.E., 2004. Risk factors involved in conception, abortion and kidding rates of goats under extensive conditions. Small Rumin. Res. 55, 191-198. 
Osuagwuh, A.I.A., 1992. Effects of strategic feed supplementation during pregnancy on birth weight and perinatal survival of West African Dwarf kids. J. Agric. Sci., Camb. 119, 123-126.

Parr, R.A., Cumming, I.A. \& Clarke, I.J., 1982. Effects of maternal nutrition and plasma progesterone concentrations on survival and growth of the sheep embryo in early gestation. J. Agric. Sci., Camb. 98, 39-46.

Parr, R.A., Davis, I.F., Fairclough, R.J. \& Miles, M.A., 1987. Overfeeding during early pregnancy reduces peripheral progesterone concentration and pregnancy rate in sheep. J. Reprod. Fert. 80, 317-320.

Robinson, J.J., 1983. Nutrition of the pregnant ewe. In: Sheep Production. Ed. Haresign, W., Butterworths. pp. 111-132.

Safari, A. \& Fogarty, N.M., 2003. Genetic parameters for sheep production traits. Estimates from the literature. NSW Agriculture, Orange Agricultural Institute, Orange NSW 2800 Australia. Australian Sheep Industry Cooperative Research Centre. pp. 13-21.

SAS, 2004. Statistical Analysis Systems user's guide (Version 9.1). SAS Institute Inc., Raleigh, North Carolina, USA.

Snyman, M.A., 2007. Body weight and growth rate of South African Angora goat kids under different preand post weaning management systems. S. Afr. J. Anim. Sci. 37, 132-141.

Terblanché, E.leF., 1988. 'n Ondersoek na Angorabokboerdery in die Republiek van Suid-Afrika. BKB, Port Elizabeth.

Van der Westhuizen, J.M., Wentzel, D. \& Grobler, M.C., 2004. Reproduction. In: Angora goats and mohair in South Africa - 2004 Revised ed. Mohair South Africa, P.O. Box 2243, Port Elizabeth 6056, South Africa.

Wentzel. D., 1982. Supplementary feeding of Angora goats. Angora Goat and Mohair J. 24 (2), 25-29.

Williams, A.H. \& Cumming, A.I., 1982. Inverse relationship between concentration of progesterone and nutrition in ewes. J. Agric. Sci., Camb. 98, 517-522. 